\title{
ASL perfusion in atypical Japanese encephalitis
}

Dear Editor,

We report an unusual magnetic resonance imaging (MRI) appearance of Japanese Encephalitis (JE) that one has to be aware of in the appropriate clinical setting. We also highlight the role of 3D Arterial spin labelling (ASL) perfusion in JE.

A 62-year-old male was admitted in emergency department at Rajiv Gandhi Government General Hospital, Madras
Medical College, Chennai, in a disoriented state with recurrent new onset seizures and fever. On examination, initially patient was restless, later he became drowsy and did not respond to oral commands. MRI was performed on 3T MR scanner (SIEMENS SKYRA). Standard Institute MRI Brain protocol was done along with 3D ASL Perfusion and Contrast study. T2-weighted images showed significant gyral edema in left fronto-parieto-temporal region and hyperintensity in left caudate, putamen and 
thalamus [Figure 1A]. T1 Weighted contrast subtracted images showed no abnormal enhancing areas in brain parenchyma [Figure 1B]. Diffusion weighted images (DWI) [Figure 2A] and apparent diffusion coefficient (ADC) [Figure 2B] showed restricted diffusion in left fronto-parieto-temporal region and left deep gray matter. ASL perfusion showed relatively increased cerebral blood flow (CBF) in left fronto-parieto-temporal region [Figure 3]. Both serum and cerebrospinal fluid (CSF) were positive for JE antibodies [Table 1]. The patient showed clinical improvement, becoming conscious, oriented and ambulant after appropriate symptomatic management.

Follow-up MRI taken after 3 weeks showed persistent gyral FLAIR hyperintensities [Figure 4] and normalization of ADC values with facilitated diffusion in left fronto-parieto-temporal region and deep grey matter [Figure 5A and B]. ASL perfusion showed normalization of CBF [Figure 6].

Japanese encephalitis (JE) is mosquito-borne flavi-viral endemic encephalitis, the diagnosis of which is based on the essential and supportive criteria. ${ }^{[1]}$

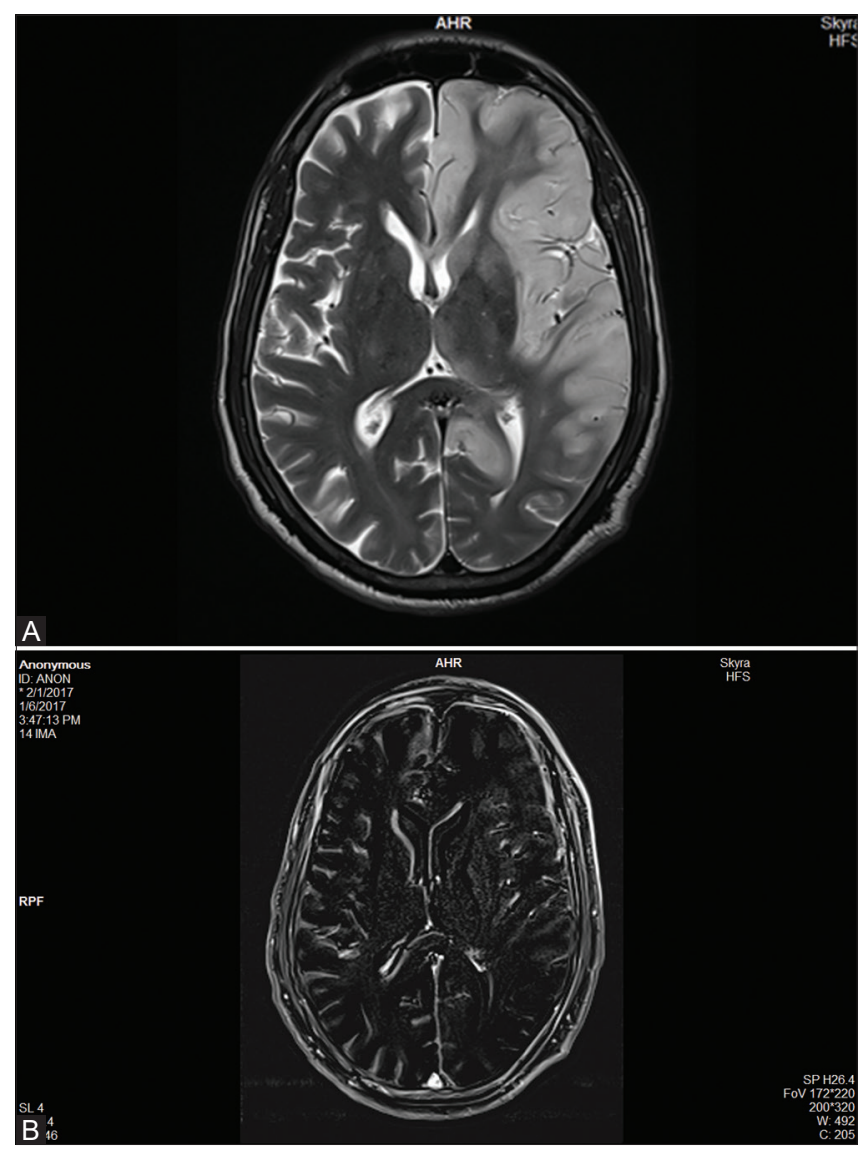

Figure 1 (A and B): (A) T2WI shows significant gyral edema in left fronto-parieto-temporal region and hyperintensity in left caudate, putamen and thalamus. (B) T1 contrast subtracted image shows no abnormal enhancing areas in brain parenchyma
Thalamic lesions on computed tomography (CT) or magnetic resonance imaging (MRI) scans in an appropriate clinical and epidemiological background is suggestive of the diagnosis.

Pathologic changes in the brains of acute JE patients are characterized by glial nodules and circumscribed necrolytic foci mainly in bilateral thalami, substantia nigra, basal ganglia, brain stem, cerebellum, cerebral cortical, and white matter ${ }^{[2]}$ and the MRI findings reflect the pathologic changes in those areas. ${ }^{[3,4]}$ Among them, the most consistent characteristic findings in JE is bilateral thalamic lesions with or without hemorrhage on MRI. ${ }^{[4]}$ However, unilateral lesions in JE have also been extremely rarely reported. ${ }^{[2]}$

Our case had unilateral involvement of left fronto-parieto-temporal region and left deep grey matter

Table 1: Serology report

\begin{tabular}{llllll}
\hline Age & Sex & Hospital & Sample & Investigation & Result \\
\hline 62 & M & RGGGH & Blood & HSV & Negative \\
& & and CSF & CMV & Negative \\
& & VZV & Equivocal \\
& & HBsAg & Negative \\
& & & EBV & Negative \\
& & & JE Serum & Positive \\
& & & JE CSF & Positive \\
\hline
\end{tabular}

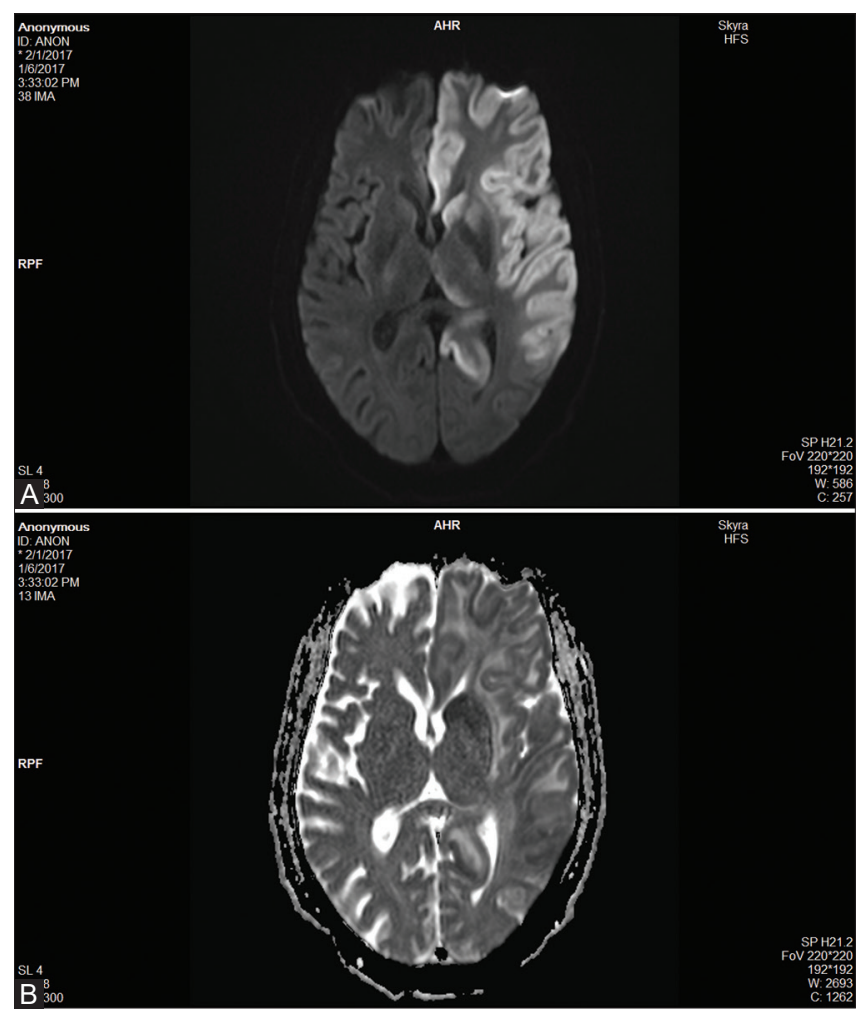

Figure 2 (A and $B$ ): (A) DWI shows diffusion restriction in left fronto-parieto-temporal region and left deep grey matter. (B) ADC shows diffusion restriction in left fronto-parieto-temporal region and left deep grey matter 


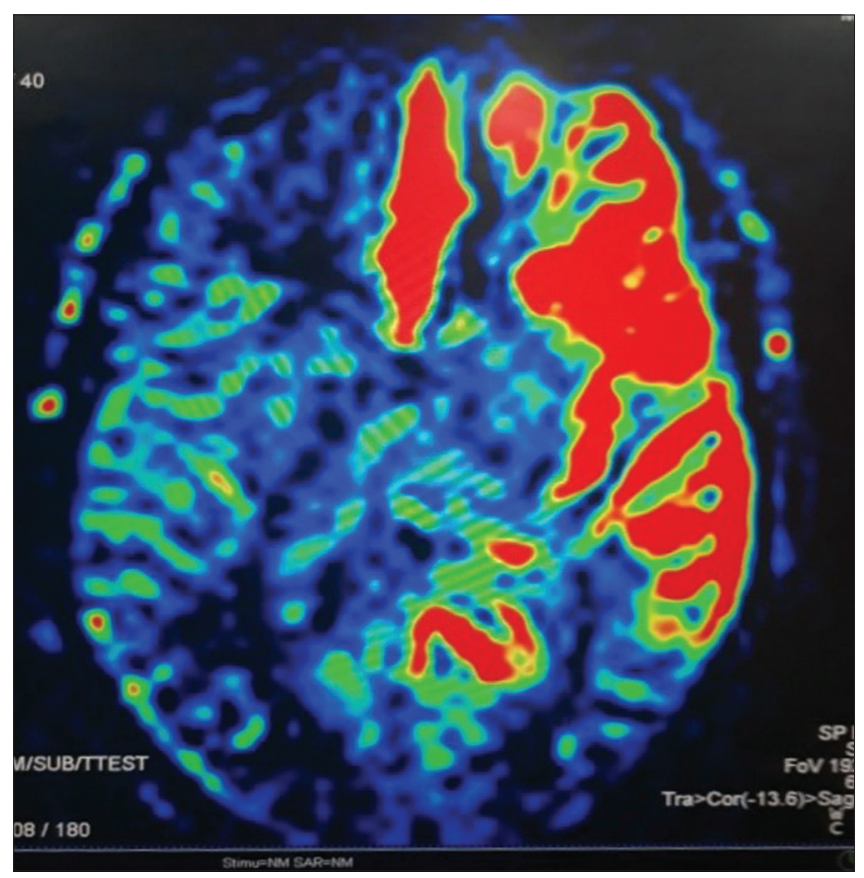

Figure 3: ASL image shows increased cerebral blood flow (CBF) in left fronto-parieto-temporal region

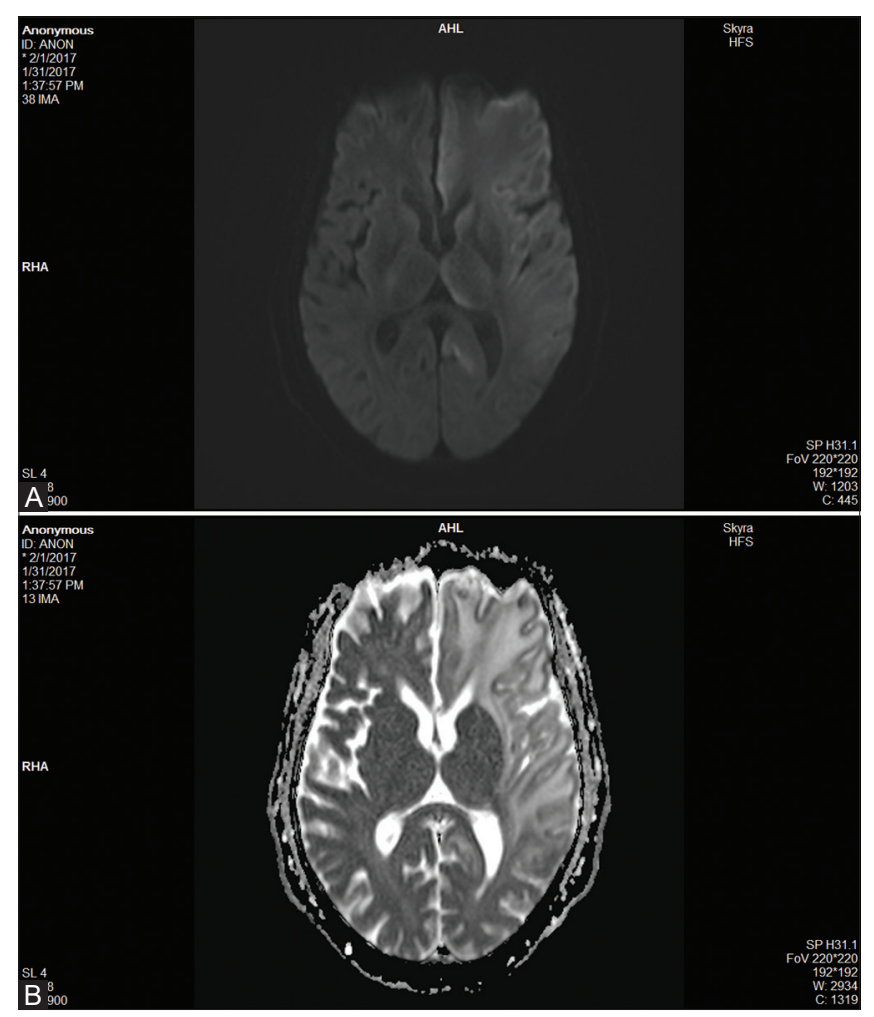

Figure 5 (A and B): (A) DWI shows facilitated diffusion in left fronto-parieto-temporal region and deep grey matter. (B) ADC shows normalization of ADC values with facilitated diffusion in left fronto-parieto-temporal region and deep grey matter

(caudate nucleus, putamen and thalamus) showing T2 hyperintensity with diffusion restriction and increased perfusion in ASL.

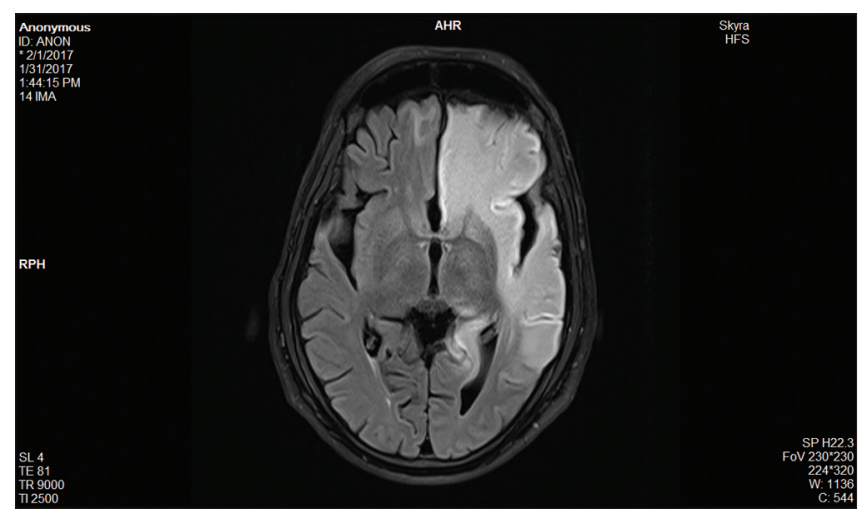

Figure 4: Fluid Attenuated Inverted Recovery (FLAIR) AXIAL images show persistent gyral edema

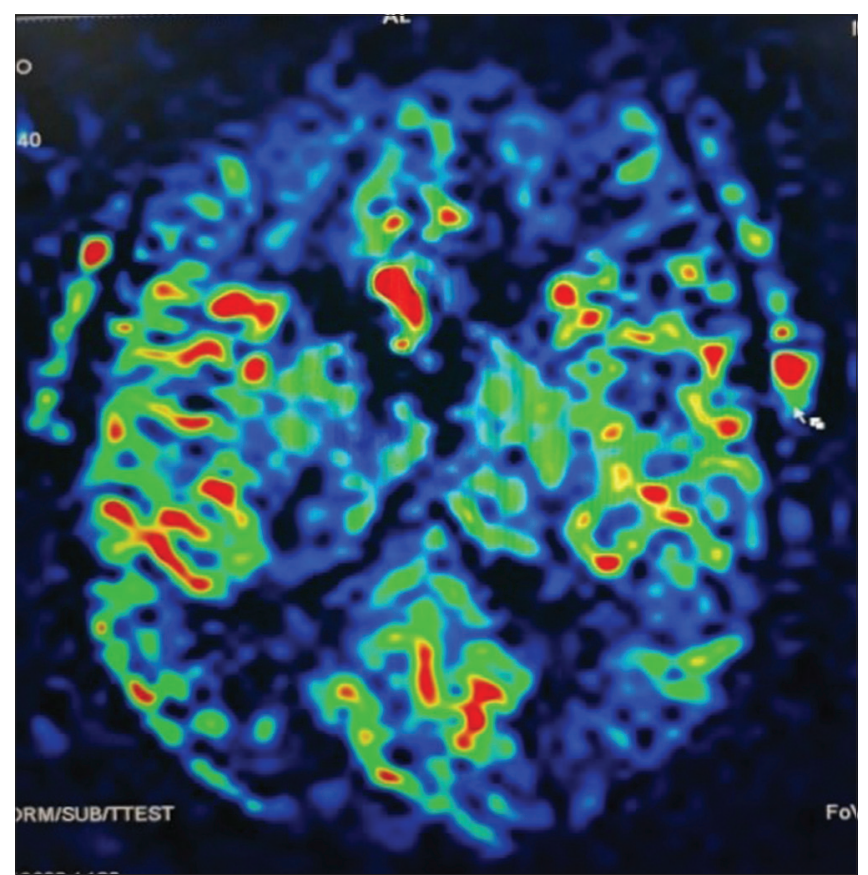

Figure 6: ASL image shows normalization of perfusion with decreased cerebral blood flow (CBF) in left fronto-parieto-temporal region and deep grey matter

Acute infarct may be considered as a possibility in view of restricted diffusion. However, for such a massive hemispherical involvement with diffusion restriction, there is no neurological deficit. The pattern also did not conform to any particular vascular territory and the MR Angiogram showed patent arteries. Although Perfusion deficits occur with acute infarcts, post-ischemic hyper-perfusion may also occur and may reflect loss of autoregulation. ${ }^{[5]}$ It may be seen in patients receiving intravenous thrombolysis and may be related to 'luxury perfusion' as seen on PET CBF imaging studies. ${ }^{[5]}$ The perfusion changes are usually more heterogeneous appearing in Stroke rather than the homogenous pattern seen with Encephalitis. Our case has increased perfusion with no neurological deficit, so possibility of infarct was considered less likely. 
Hyper perfusion in acute encephalitis is known to occur, as previously reported in ASL perfusion studies. ${ }^{[6]}$ Knowledge of CBF disturbances in Japanese encephalitis is limited. We highlight the utility of 3D ASL perfusion as a biomarker of disease activity in this particular case. It can not only be used for identifying early changes but also for follow-up as demonstrated.

99mTc-hexamethylpropyleneamine oxime (HMPAO) SPECT has been used earlier in JE evaluation. In early stages, it showed a significant increase of HMPAO uptake and in follow-up revealed decreased uptake, ${ }^{[7]}$ similar to the perfusion abnormality in our case. Similar changes with hypoperfusion in subacute or chronic cases in SPECT imaging have also been reported. ${ }^{[8]}$ However, ASL perfusion is a robust tool with added advantage of no contrast being and no radiation, compared to SPECT and can be part of MRI protocol in Encephalitis imaging, if available. ${ }^{[9]}$

We have highlighted the utility of 3D ASL perfusion as a biomarker of disease activity in this particular case. It can not only be used for identifying early changes but also for follow-up as demonstrated. To our knowledge, no reports of ASL Perfusion being used to follow-up JE patients have been found.

\section{Declaration of patient consent}

The authors certify that they have obtained all appropriate patient consent forms. In the form the patient(s) has/have given his/her/their consent for his/her/their images and other clinical information to be reported in the journal. The patients understand that their names and initials will not be published and due efforts will be made to conceal their identity, but anonymity cannot be guaranteed.

\section{Financial support and sponsorship}

Nil.

\section{Conflicts of interest}

There are no conflicts of interest.

\section{Babu Peter Sathyanathan,} Aishwarya Ravichandran, Ravi Ranganathan Barnard Insitute of Radiology, Madras Medical College, Chennai, Tamil Nadu, India. E-mail: drbabupeter@gmail.com

\section{References}

1. Basumatary LJ, Raja D, Bhuyan D, Das M, Goswami M, Kayal AK. Clinical and radiological spectrum of Japanese encephalitis. J Neurol Sci 2013;325:15-21.

2. Handique SK, Das RR, Barman K, Medhi N, Saharia B, Saikia P, et al. Temporal lobe involvement in Japanese encephalitis: Problems in differential diagnosis. AJNR Am J Neuroradiol 2006;27:1027-31.

3. Kalita J, Misra UK. Comparison of CT scan and MRI findings in the diagnosis of Japanese encephalitis. J Neurol Sci 2000;174:3-8.

4. Kumar S, Misra UK, Kalita J, Salwani V, Gupta RK, Gujral R. MRI in Japanese encephalitis. Neuroradiology 1997;39:180-4.

5. Yu S, Liebeskind DS, Dua S, Wilhalme H, Elashoff D, Qiao XJ, et al. Post ischemic hyperperfusion on arterial spin labeled perfusion MRI is linked to hemorrhagic transformation in stroke. J Cereb Blood Flow Metab 2015;35:630-7.

6. Noguchi T, Yakushiji Y, Nishihara M, Togao O, Yamashita K, Kikuchi $\mathrm{K}$, et al. Arterial spin-labeling in central nervous system infection. Magn Reson Med Sci 2016;15:386-94.

7. Kimura K, Dosaka A, Hashimoto Y, Yasunaga T, Uchino M, Ando M. Single-photon emission CT findings in acute Japanese encephalitis. AJNR Am J Neuroradiol 1997;18:465-9.

8. Misra UK, Kalita J, Srivastav A, Pradhan PK. The prognostic role of magnetic resonance imaging and single-photon emission computed tomography in viral encephalitis. Acta Radiological 2008;49:827-832.

9. Yakushiji Y, Kurohara K, Tanaka A, Kuroda Y, Uchino A. A case of Japanese encephalitis presenting with unilateral lesions in diffusion-weighted MRI. Rinsho Shinkeigaku 2001;41:602-5.

This is an open access journal, and articles are distributed under the terms of the Creative Commons Attribution-NonCommercial-ShareAlike 4.0 License, which allows others to remix, tweak, and build upon the work non-commercially, as long as appropriate credit is given and the new creations are licensed under the identical terms.

\begin{tabular}{|l|l|}
\hline \multicolumn{2}{|c|}{ Access this article online } \\
\hline Quick Response Code: & \\
\hline & Website: \\
\hline
\end{tabular}

Cite this article as: Sathyanathan BP, Ravichandran A, Ranganathan R. ASL perfusion in atypical Japanese encephalitis. Indian J Radiol Imaging 2020;30:536-9.

Received: $18-\mathrm{Apr}-2020$

Accepted: 02-Sep-2020

Revised: 16-May-2020

Published: 13-Jan-2021

๑ 2021 Indian Journal of Radiology and Imaging | Published by Wolters Kluwer - Medknow 